BNL- -43322

DE $90 \quad 002311$

\title{
RF Stability, Control and Bunch Lengthening in Electron Synchrotron Storage Rings
}

\author{
J. M. Wachtel \\ National Synchrotron Light Source \\ Brookhaven National Laboratory \\ Upton, New York 11973
}

Abstract

\begin{abstract}
A self-consistent theory for nonlinear longitudinal particle motion and RF cavity excitation in a high energy electron storage ring is developed. Coupled first order equations for the notion of an arbitrary number of particles and for the field in several RF cavities are given in the form used in control system theory. Stochastic quantum excitation of synchrotron motion is included, as are the effects of RF control system corrections. Results of computations for dourle cavity bunch lengthening are given.

*Work perfoimed under the auspices of the U.S. Department of Energy.

${ }^{+}$On leave from Elta Electronics Industry, Ashdod, Israel.
\end{abstract}




\section{Introduction}

Several later generation synchrotron radiation facilities are now about to be built. Three that pigure prominently are ESRF at Grenoble, APS at Argonne and ALS at Berkeley. Designed to provide radiation intensities orders of magnitude greater than existing facilities, they will place demanding requirements on the brightness of the electron or positron beams they nust store. At the high particle densities and bean currents to be achieved, phenomena that degrade beam brightness and beam lifetime will be nore pronounced. There are many troublesome effects [Ref. 1], including for example, high frequency instability in the distributed impedance of the vacuun ring, coupled bunch instability, and scattering between particles within a bunch.

An effective way to ameliorate then all is to store the circulating particles in flat bottoned nonharmonic RF potential buckets. Particle bunches are thereby lengthened and the particle density is reduced. As a result high, frequency wake fields are attenuated and the frequency of particle collisions is reduced. Coherent synchrotron oscillations are dephased in the nonharmonic potential, damping coupled bunch modes.

The harmonic covity method [Refs. 2,3] used to create quartic potential buckets is not particularly new. Although it has been demonstrated in low current fings it has not yet been used in high intensity electron rings for a significant reason. The reaction of the bean on the cavities supporting the accelerating fields may be so strong that arbitrary specification of the fields is technically difficult. The harmonic cavity method requires precise control of the phase and amplitude of the ield in a double cavity system. This has not yet been achieved for cavities that are strongly coupled to the bean. In principle, the nethod ought to work and it should becone a standard element of synchrotron storage ring design. What is needed is a framework for understanding its problems and designing a system with the necessary elenerits of control. That is the objective of the research described in this report and of the earlier work [Ref. 4] superceded by it.

The theory of particle bunches interacting with resonant structures in a ring has its beginning early in the history of high energy storage ring development. Rigid bunch dynamics in harmonic RF potentials are well understood. The benchmark of this work is the ubiguitous Robinson instability. Later, the behavior of particles trapped in harmonic RF potential buckets was studied using kinetic theory. Fokker-Planck equations were solved to describe particle diffusion but without self consistently coupling the RF cavity fields to the particle motion. Solutions of the Fokker-Planck equation for particles in fixed non-harmonic potentials were also achieved [Ref. 5]. 
Past theoretical work has treated rigid bunches interacting with compliant cavities on the one hand, and rigidly excited cavities interacting with freely diffusing particles on the other. Attempts at developing a self-consistent theory allowing for both are rare [Ref. 6]. Moreover, any self-consistent theory is likely to use linear perturbation theory, eliminating from consideration large amplitude synchrotron oscillations, nonlinear RF potentials, and discrete time corrections in the storage $r$ ing RF control system.

For the harmonic cavity ring all of the interactions between the RF systen and the particles have to be considered. Diverse effects such as quantum excitation of synchrotron oscillations, changes in the phase and voltage of the RF generator signals driving the cavities, and fields induced in the cavities by the particles must all be included. The task is to develop a set of equations that describe them all acting together. The system that will be treated in this paper is restricted to be an ideal, one dimensional storage ring with narrow band resonant cavities. Transverse interactions will not be considered. Any number of cavities with resonant frequencies near harmonics of the particle rotation frequency may be involved and the motion of the particles will be followed individually in an arbitrarily large simulacion sample.

The governing equations are a set of non-linear, coupled first order differential equations written in a format used in multi-variate control system theory. In addition to being convenient for progamming a computer solution, the format provides a systematic method for introducing noise to simulate quantum excitation due to photon emission. The effects of statistical errors in time sampled measurements and discrete control system corrections are easily introduced. The equations attempt to describe all of the phenomena related to - longitudinal synchrotron motion occurring on a time scale that is long in relation to the period of particle rotation.

Equations of motion for a particle interacting with several cavities, as well as equations for the field of a cavity due to the motion of the particles are described in the following section. They form the basis for a multi-dimensional set of coupled equations to be used in a computer sinulation. Before proceeding to that, the equations for one rigid bunch (or macro-particle) interacting with one cavity will be linearized and a mode analysis vill be performed in section 3 . Although it vill show standard results, certain relationships will be clarified, for example the parameters that force the Robinson instability to become monotonic growth. 
Spectral theory of random processes is applied in section 4 to include the effect of synchrotron radiation photon statistics. Brownian motion of the particles in synchrotron phase space is the resulting randon process. Computations are presented to demonstrate the statistica? accuracy of this step. Section 5 shows a complete set of equations written in the state function form of control syster theory. Computed results depicting the time development of particle and field states are shown in the final section. Discrete time corrections, such as might actually occur in a system controlled by a central processor able to receive measurements and issue instructions to the RF system, are used in a simulation of bunch lengthening in a high current beam. 


\section{Coupled Equations}

In his well known review of the basic theory of electron storage rings [Ref. 7] $M$. Sands wrote the equations for longitudinal particle motion directly in reduced form. His starting point was the assumption that there is a synchronous longitudinal position at which a particle with the storage ring design energy $E_{0}$ will execute perpetual unperturbed rotation at constant period $\mathrm{T}_{0}$. This rapid rotation is removed by writing the particle coordinate $\tau$ with respect to the synchronous position. When its energy deviates from $E_{0}$ the particle position must slip at a rate proportional to the energy difference. Defining the position $T$ in units of time of travel at the speed of light, and the energy difference $\epsilon$ in the sane units as $E_{0}$

$$
d \tau / d t=-\alpha \epsilon / E_{O}
$$

This is the position equation of motion. The proportionality factor $\alpha$ called the momentum compaction is a feature of the magnetic lattice of the storage ring. It is a small positive number in relativistic electron rings.

A circulating particle loses a quantum of energy at each emission of synchrotron radiation. The impulse is sudden and essentially anti-parallel to the particle momentum. These events occur at statistically distributed intervals and with statistically distributed photon energies that depend upon the particle energy and the curvature of its orbit. Since many photons are enitted during one rotation around the ring and since the cumulative energy loss is much smaller than $E_{O}$, detailed knowledge of each event is unnecessary. Moments of the photon emission statistics suffice to relate photon .emission to the particle orbits.

Classical synchrotron radiation theory accounts for the average energy loss. Following Sands, the classical energy radiated by a particle at energy $E_{0}+\epsilon$ during one revolution is written $U_{0}+D E$. The term $D \in$ represents the first order energy dependence of the classical enission rate. But in quantum terns this can only represent the expected energy loss. If a zero mean randon process $N(t)$ is added to the classical radiation rate it will generate statistical dispersion. Statistical dispersion must accompany quantum emission. This random process should have spectral properties that reproduce the statistics of synchrotron photon enission. 
Accordingly the radiation loss rate may be written

$$
(d \epsilon / d t)_{\operatorname{rad}}=-\left(U_{O}+D \epsilon\right) / T_{O}+N(t) .
$$

The term $D E$ causes coherent damping of synchrotron oscillations. It also limits particle diffusion in longitudinal phase space due to the fluctuating force $N(t)$.

Energy is restored to circulating particles as they cross gaps in RF cavities gaining energy increments eV(t). If a gap is short in relation to the RE wavelength $e V(t)$ will be close to the instantaneous electric potential across it. Storage ring cavities are usually made that way so the guasi-static approximation is assumed to be valid. $V_{m}(-\tau)$ is identified

with the gap potential at the mth cavity at the instant a particle at position $\tau$ crosses it. For one particle in a ring having several RF cavities

$$
d \epsilon / d t=\left[\sum_{m} e V_{m}(-T)-U_{0}-D E\right] / T_{0}+N(t) .
$$

This is the momentum equation for longitudinal particle motion. Both $\epsilon(t)$ and $\tau(t)$ are slowly varying functions of $t$ ime.

RF cavities used in high energy electron storage rings are generally high $Q$ narrow band resonators with short capacitive gaps to concentrate the longitudinal electric field at the particle orbit. The resonant frequency of the operating mode is near but not necessarily equal to a harmonic of the particle rotation frequency. Let the resonant frequency of the fundanental cavity equal $\omega_{0}+\Delta \omega$, where $\omega_{0} T_{0} / 2 \pi$ is an integer equal to the number of particle buckets in the $r$ ing. There may be more than one fundamental cavity but all the others are resonant near integer multiples of $\omega_{0}$. Although the cavities may be detuned, all are forced to oscillate in near perfect synchronism at harmonics in $\omega_{0}$. Departures from synchronism will, however, occur on a slow time scale determined by the cavity bancluidth and the particle drift. Equations for the slow variation of the cavity field are given below. 
A full derivation of the following results is given in Appendix I. The vector potential field may be written

$$
\bar{A}(r, t)=a(t) \sin \left[\omega_{0} t+\eta(t)\right] \bar{u}(r)
$$

where $a(t)$ and $\eta(t)$ are anplitude and phase functions varying slowly in time. Similarly, the gap potential may be written

$$
v(r, t)=v(t) \cos \left[\omega_{c} t+\eta(t)\right] .
$$

Defining a gap capacitance $C$ giving the RF energy stored in the cavity $\mathrm{Cv}^{2} / 2$, and refering the generator driven RF current to the impedance across the gap,

$$
I_{g}(t)=I_{g} \cos \left(\omega_{0} t+\eta_{g}\right)
$$

equations for the slowly varying gap voltage and its phase are

$$
\dot{v}+\left(\omega_{0} / 2 Q\right) v=\left(e / \mathrm{CT}_{0}\right) \sum_{j} \cos \left(-\omega_{0} \tau_{j}+\eta\right)+\left(I_{g} \cdot 2 \mathrm{C}\right) \cos \left(\eta-\eta_{j}\right)
$$

and

$$
v(\dot{\eta}-\Delta \omega)=-\left(e / C T_{0}\right) \sum_{j} \sin \left(-\omega_{0} \tau_{j}+\eta\right)-\left(I_{g} / 2 C\right) \sin \left(\eta-\eta l_{g}\right)
$$

A dot indicates differentiation with respect to time. Each particle (an electron carrying charge e) contributes to the beam current through the sum. Dissipative currents in the cavity wall as well as the coupled generator resistance are included in the loss term $\omega_{0} v / 2 Q$ while dispersion due to cavity detuning and coupled generator reactance is included in the term $-v \Delta \omega$. The generator current $I_{g}$ and its phase $\eta_{g}$
are constants.

Eqs. (1), (2), (3), and (4) repeated for each particle and each cavity (modified to its correct harmonic) constitute a complete description of the slow time scale behavior of the longitudinal particle motion and the cavity fields. Each cavity will have a different set of constants $C, Q, I_{g}, \eta_{g}$, and
$\Delta \omega$. 


\section{Linear Modes}

Miyahara, et al [Ref. 8] used a second harmonic cavity in the SOR electron storage ring and succeeded to store electrons at an inflection point on the total acceleration field. They observed bunch lengthening. They also observed the tendency of the system to run away from the bunch lengthened state. Interaction between the bean and the harmonic cavity changed the phase of the field and simultaneously shifted the synchronous position of an electron bunch. Miyahara, et al call the effect an "equilibrium phase instability".

What they observed is a special case of the Robinson instability occuring when the frequency for small amplitude synchrotron oscillations vanishes. The following linear mode analysis for one rigid particle bunch interacting with a harmonic cavity will show the relationship. It is performed on four slowly varying state variables $V(t), \eta(t), \epsilon(t)$, and $\tau(t)$. Growth rates for small departures from equilibrium states and the mode structure are given.

The problem to be analyzed involves a nth harmonic cavity excited by the beam and a funidamental cavity excited only by an external RF generator. The harmonic cavity may also have a component excited by a generator which will be considered part of the fundamental field waveforn, a permitted procedure because only the slope of the generator driven field appears in the linearized equations of motion. For a $r$ igid bunch there is no statistical dispersion due to photon emission.

Using dimensionless variables, let $v(t)$ and $\gamma(t)$ be the anplitude and phase of the potential generated by the beam in the harmonic cavity gap. RF potentials are normalized to $U_{0}$. Time $t$ as well as the synchrotron coordinate $\tau(t)$ are expressed in radians at the angular frequency $\omega_{0}$. The energy - $E(t)$ of each electron is measured in units $E_{o} / \alpha$. Dropping any notation to indicate this normalization and using a prime to indicate differentiation with respect to radian time, the four equations governing the system are written

$$
\begin{aligned}
& v^{\prime}=-v(m / 2 Q)+A \cos (-m \tau+\eta), \\
& v \eta^{\prime}=v(m / 2 Q) \tan \psi-A \sin (-m \tau+\eta), \\
& \tau^{\prime}=\epsilon, \\
& \epsilon^{\prime}=-B\left[v_{g}(-\tau)+v \cos (-m \tau+\eta)-1\right]-d \epsilon .
\end{aligned}
$$


where $A=-I_{b} e / C U_{0} \omega_{0}$,

$$
\begin{aligned}
B & =\alpha U_{0} / E_{0} \omega_{0} T_{0} . \\
\tan \psi & =2 Q \Delta \omega / m \omega_{0} .
\end{aligned}
$$$$
d=D B / \alpha \text {, and }
$$
The detuning angle of the harmonic cavity $-\frac{\pi}{2}<\psi<\frac{\pi}{2}$, and $I_{b}$
is the average current of the beam.

There is an equilibrium state with

$$
v^{\prime}=\eta^{\prime}=\epsilon^{\prime}=\epsilon=\gamma=0 \text {. }
$$

In that state the bunch remains at rest at the synchronous phase while

$$
\begin{gathered}
v=-2 A Q \cos \psi / m, \eta=\psi+\pi, \text { and } \\
V_{g}(0)+V \cos \eta=1 .
\end{gathered}
$$

The linear mode analysis is performed for small perturbations around this equilibrium state. Note that the phase of the field excited by the $b=a m$ is the detuning angle rotated through $\pi$ radians because the field withdraws energy from the beam. For the same reason $A<0$.

Assuming that the small perturbstions $\delta v, \delta \eta, \epsilon$, and $\tau$ vary as $\exp \left(s \omega_{0} t\right)$, the linearized equations of motion are

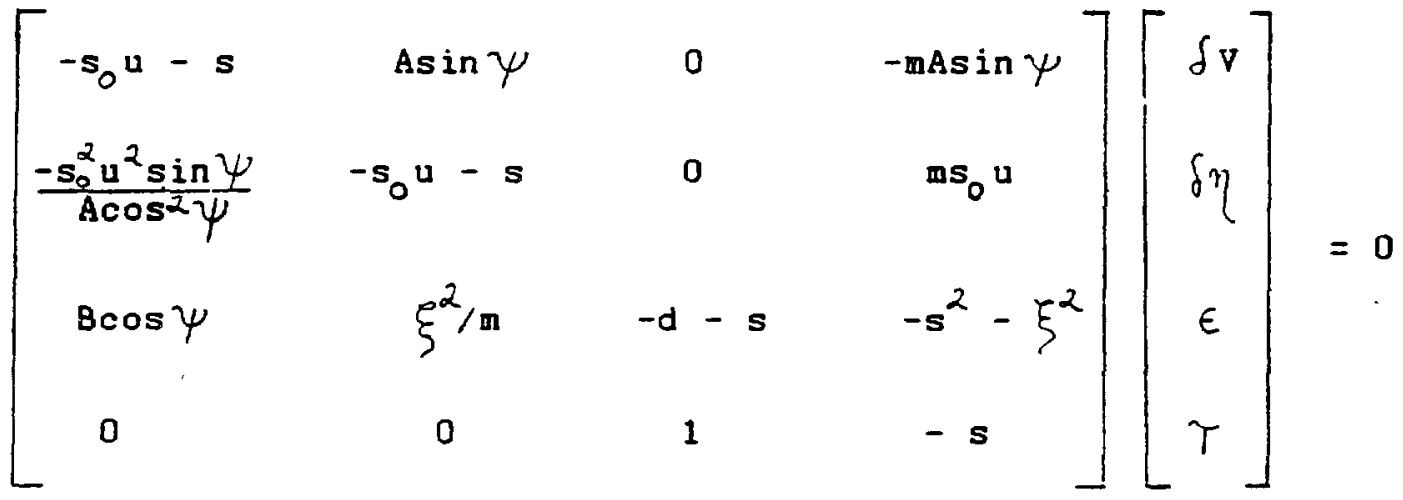

where

$$
s_{0}^{2}=-B V^{\prime}(0), \quad \xi^{2}=B A Q \sin 2 \psi .
$$

and

$$
u=m / 2 Q s_{0} \text {. }
$$


Eigenvalues of $\mathbf{s}$ satisfy

$$
\begin{aligned}
\left(s^{2}+s d+s_{0}^{2}+\xi^{2}\right)\left(s^{2}+2 s_{0} u s\right. & \left.+s_{0}^{2} u^{2} / \cos ^{2} \psi\right) \\
& -\left(s_{0}^{2} u^{2} \xi^{2} / \cos ^{2} \psi\right)=0 .
\end{aligned}
$$

The real constant $s_{0}$ is the normalized value of the angular frequency for small amplitude synchrotron oscillations in the generator driven field.

As $I_{b}$ increases, positive and negative frequency synchrotron modes couple to the evanescent cavity modes. To see the development of the mode interaction for low beam current, the eigenvalue equation may be expanded around each of the uncoupled solutions assuming that $|\xi / s| \ll 1$. After all the straightfoward algebra is done the normalized complex frequencies for the weakly coupled modes may be written

$$
\begin{aligned}
& s_{1}=s_{2}^{*}=-d / 2+i s_{0}-\left(\xi^{2} / 2 s_{0}\right)(F+i G) / J \\
& s_{3}=s_{4}^{*}=u s_{0}(-1+i \tan \psi)-\left(\xi^{2} / 2 u s_{0}\right)(H+i R) / J
\end{aligned}
$$

where

$$
\begin{aligned}
& \mathbf{F}=2 \mathbf{u}^{3}\left(1+\tan ^{2} \psi\right), \quad \mathbf{H}=-\mathbf{F u}, \\
& \mathbf{G}=(F / 2 u)-4 \mathbf{u}^{2}-1, \\
& \mathbf{R}=(F / 2 u)\left[u^{2}\left(1-\tan ^{2} \psi\right)+1\right] / \tan \psi, \text { and } \\
& J=\left[u^{2}\left(1-\tan ^{2} \psi\right)+1\right]^{2}+\left[2 u^{2} \tan \psi\right]^{2} .
\end{aligned}
$$

The unperturbed synchrotron modes are very weakly damped. When the harmonic cavity is resonant above a $\omega_{0}$ the real term involving $F$ may dominate the coherent damping term $d / 2$, even For a beam current that is rather small. This is the Robinson instability. The unperturbed cavity modes are strongly damped. When the harmonic cavity is resonant below m $\omega_{0}$ the synchrotron modes are further stabilized while the cavity modes are destabilized by an equal amount. Nevertheless these modes remain stable for all values of $I_{b}$. 
When $I_{b}$ is large the bunch and the cavity are strongly coupled aid the expansion used for small $\xi / s_{0}$ is invalid. It is also invalid for a quartic equilibriun potential because so would in that case vanish. Roots of the eigenvalue equation must then be evaluated numerically. Two additional parameters, the detuning angle $\psi$ and the damping factor $u$, determine the location of the roots in the complex s-plane. For many storage rings the synchrotron oscillation frequency $s_{0} W_{0}$ is comparable with the cavity damping damping constant $\omega_{0} / 2 Q$. Therefore $u$ is of the order of one. Figures $1 \mathrm{a}$ and $1 \mathrm{~b}$ show how the roots move in the complex plane as the coupling parameter grows for fixed values of $u$ and $\psi$. Note that the real part of $s$ is negative for negative tuning. All the modes are stable. For positive tuning the two modes that evolve from the pure synchrotron modes are unstable. The coherent danping is negligible. At some value of $I_{b}$ two or four roots are real. The oscillations cease and give way to monotonic growth or decay.

Having evaluated the mode eigenvalues the mode structure is obtainert by substituting them for $s$ in the equations of motion. The cooperative motion of $\delta v, \delta \eta, \epsilon$, and $\tau$ may be expressed in terms of any one. Choosing $\tau$ the nodes are written

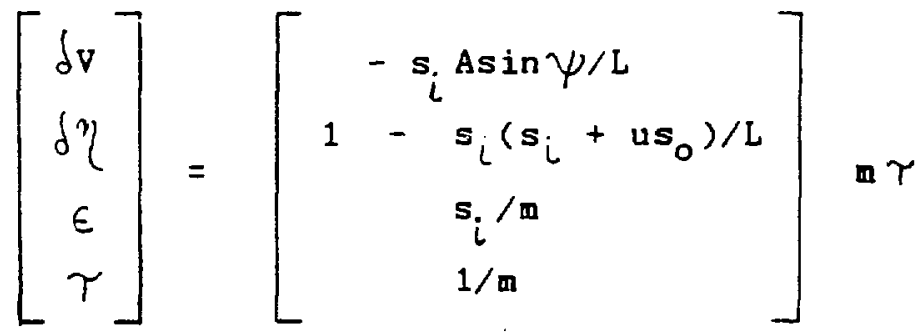

where

$L=\left(s_{i}+u s_{0}\right)^{2}+\left(u s_{0} \tan \psi\right)^{2}$

and

$s_{i}=s_{1}, s_{2}, s_{3}$, or $s_{4}$.

The analysis of rigid bunch linear modes is not pursued further. 


\section{Photon Emission}

The random process $N(t)$ was put into Eq.(2) to produce phase space diffusion. Properly determined, $N(t)$ will have the same effect as random photon emission. Now it is necessary to derive a useful form for it.

Several fundamental assumptions precede the derivation. First, the r.m.s. energy deviation for electrons executing small amplitude synchrotron oscillations is a priori known in terms of the magnetic lattice of the storage ring. The classical radiation formulae are used to infer the average photon emission rate. Given the fact that the emission may occur at any phase of a synchrotron oscillation the r.m.s. energy deviation from $E_{0}$ follows as

$$
\sigma_{\epsilon}=\left(u_{C} E_{0}\right)^{\frac{1}{2}} \text {, }
$$

where $v_{c}$ is the critical synchrotron photon energy as determined by $E_{0}$ and the geometry of the storage ring. A derivation of this result is given by sands [Ref, 7].

Second, photon emission statistics and consequently $N(t)$ are independent of the the slowly varying features of the particle motion. Suppose that a certain random process $N(t)$ adequately simulates the photon emission for one RF system. Then it is assumed that it is equally valid for any other RF system installed in the same storage ring lattice.

Consider a storage ring with one RF cavity excited to a high field amplitude by an RF generator. Suppose the beam current is so low that the perturbation of the cavity field is negligible. Stored electrons will then execute simple harmonic synchrotron oscillacions excited by photon emission noise in the same stationary RE potential, independently of one another. Each electron is one member of an ensemble of noise driven harmonic oscillators obeying the equation of motion

$$
\tau^{\prime \prime}+d \tau^{\prime}+s_{0}^{2} \tau=N(t)
$$

Random processes $N(t)$ for different electrons have identical probability distributions iu are completely uncorrelated. If $N(t)$ is a stationary random process, then its autocorrelation function $\left\langle N\left(t^{\circ}\right) N\left(t^{\circ}+t\right)\right\rangle$ computed by ensemble averaging will be identical with the autocorrelation function $R_{N}(t)$ obtained by averaging over tine. 


$$
R_{N}(t)=\operatorname{Lim}_{T \rightarrow \infty} \frac{1}{T} \int_{0}^{T} N\left(t^{\prime}\right) N\left(t^{\prime}+t\right) d t^{\prime} .
$$

Furthermore, the Weiner-Khinchine relation states that the spectral power density $S_{M}(\Omega)$ of the process $N(t)$ is the Fourier transform of its autocorrelation function $R_{N}(t)$. Then $S_{\gamma}(\Omega)$ the spectral power density of $\tau$ follows from the Eq. (5) above.

$$
s_{\tau}(\Omega)=\frac{s_{N}(\Omega)}{\left(-\Omega^{2}+i d \Omega+s_{0}^{2}\right)\left(-\Omega^{2}-i d \Omega+s_{0}^{2}\right)}
$$

Simply interpreted, this expression states that the photon noise is filtered by a mechanical bandpass filter with a narrow resonance at the synchrotron frequency $s_{0}$. The filter output is the random process $\tau$.

The ensemble average value of $\tau^{2}$ is required. The autocorrelation function $R_{\uparrow}(t)$ is the inverse Fourier transform of $S_{\gamma}(\Omega)$. $R_{\gamma}(0)$ is the time average of $\tau^{2}$. Because the filtered random process is also stationary, $R_{\tau}(0)$ is its ensemble average as well. Reference [9], giving a clear tutorial development of the theory of random processes and linear filtering, explains this procedure in detail.

Quantum excitation is the result of a randon series of impulses due to photon enission. The impulses have essentially infinitesimal duration and occur at a rate much higher than the synchrotron oscillation frequency. The relative rates for the NSLS VUV ring are typical. One synchrotron oscillation period

is about $3.3 \times 10^{4}$ radians (angular time at the RF frequency

$52.88 \mathrm{MHz}$ ), while the coherent damping time is $1.5 \times 10^{6}$. One revolution is only $18 \pi$ radians. Nevertheless during one revolution between 10 and 100 energetic photons are enitted by each electron and many more below the ultraviolet region. The average energy radiated during one revolution is $14 \mathrm{ReV}$ and the critical photon energy is around $1 \mathrm{KeV}$. The photon noise spectrum is therefore very broad. Only a low frequency filtered segment emerges as particle motion. Were it not for the harmonic potential the filtered motion would be classical Brownian motion. Instead the electrons are confined and, apart from statistical fluctuations, tend to fill the potential well until the diffusiol. driven by randon emission is balanced by the friction of coherent synchrotron oscillation damping. 
Before proceeding to compute the diffusion it is necessary to define the randon process $N(t)$. To be a useful representation it will have zero mean and its autocorrelation function will decay in a short time. Therefore it will have a broad spectrum. Since the high frequency part of its spectrum does not pass the synchrotron oscillation filtering process, the statistical features of the particle motion will be insensitive to the exact form of $N(t)$ and in particular tc the upper cutoff frequancy of its spectrum. The magnitude of $N(t)$ must be adjusted to refroduce the r.m.s. energy deviation

$$
\sigma_{\tau}=\sigma_{\epsilon} / s_{0}
$$

There are many choices for $N(t)$ that will satisfy these requirements. A particularly simple one, well adapted to programing the equations of notion, is the constant period raniom step. A computation may be interrupted at regular intervals to re-assign random constants for the values of $N(t)$. Its autocorrelation function is a triangular pulse.

$$
R(t)=\left\{\begin{array}{cl}
\sigma_{N}^{2}\left(1-\left|t / t_{N}\right|\right), & \left|t / t_{N}\right|<1 \\
c, & \left|t / t_{N}\right|>1
\end{array} .\right.
$$

The probability distribution for the values of $N(t)$ has to give zero mean and variance $\sigma_{N}^{2}$. In other respects it may be arbitrarily chosen. The interval $t_{N}$ should be much smaller than $1 / s_{0}$.

The spectral power density of $N(t)$ follows immediately as the Fourier transform

$$
S_{N}(s .)=t_{N} \sigma_{N}^{2}\left[\frac{\sin \left(\Omega t_{N} / 2\right)}{\Omega t_{N} / 2}\right]^{2}
$$

Substituting this formula for $S_{N}(\Omega)$ in Eq. (6) and calculating the inverse Fourier transform of $S_{\gamma}(\Omega)$ leads immediately to $R_{\tau}(0)$ and hence to

$$
\sigma_{\tau}^{2}=\sigma_{N}^{2} t_{N} / 2 s_{0}^{2} d
$$

Now the probability distribution for $N(t)$ must be specified. If a uniform distribution of values between equal positive and negative limits is chosen, its variance is 


$$
\sigma_{N}^{2}=\frac{1}{3} N_{0}^{2}
$$

$N_{0}$ is the limit. By Eqs. (7), (10) and (11)

$$
N_{0}=\left(6 d / t_{N}\right)^{\frac{1}{2}} \sigma_{\epsilon} \text {. }
$$

The equations of motion including random quantum excitation are now complete.

Any probability distribution giving the correct variance and zero mean is admissible. Of course, a random process $N(t)$ that accurately represents the distribution of photon energies and the timing of emission events would be prefered. A GaussMarkov process could have been specified. Its correlation function decays exponentially. Alternatively, white noise may be used. Its spectrum is absolutely flat. The uniformly distributed random step defined by Eqs. (8), (9), (11), and (12) will be used from now. It will be used with arbitrary RF potentials.

Before exercising the complete set of equations, the motion of a large number of simulation particles will be computed in two simple stationary RF potential fields:

(I) a quadratic potential

$$
v=v_{1} \cos \left(-\tau+\eta_{1}\right)
$$

where $v_{1} \cos \eta_{1}=1$,

or (II) a quartic potential

$$
\left.\nabla=\nabla_{1} \cos i-\tau+\eta_{1}\right)+\nabla_{m} \cos \left(-m \tau+\eta_{m}\right)
$$

where

$$
\begin{aligned}
& v_{1} \cos \eta_{1}+v_{m} \cos \eta_{m}=1, \\
& v_{1} \sin \eta_{1}+m v_{m} \sin \eta_{m}=0 . \\
& v_{1} \cos \eta_{1}+m^{2} v_{m} \cos \eta_{m}=0 .
\end{aligned}
$$

and

Small amplitude oscillations in (I) are harmonic. The field varies linearly around the synchronous phase. In (II) the field has an inflection point at the synchronous phase. The frequency starts at zero and increases with oscillation amplitude [Ref. 2]. 
Computations vere performed for 512 simulation particles initially at $\epsilon=\tau=0$. The value of $V_{1}$ (normalized to $U_{0}$ ) was 5.5 wile the harmonic cavity anplitude and the phases were determined by the above relations. The harmonic number mas four. The amplitude of the random step corresponded to

$\sigma_{E}=1 \mathrm{HeV}$ and its period was $1 \times 10^{3}$ radians. Phase plots at early times (less than 20 randon step periods) show diffusion along the energy axis cccurring rapidly while rotation at the synchrotron frequency slowly mixes the energy diffusion with

diffusion along $\tau$. By $t=5 \times 10^{5}$ the cross correlation between $\varepsilon$ and $\gamma$ was negligible with the particles filling an erect phase space ellipse. Smoothed histograms for the energy and displacement of 512 particles in both potentials are shown in Figs. 2a,b. Energy diffusion is faster in the quartic potential, in agreement with Hofmann and Myers [Ref. 2].

At $t=2 \times 10^{6}$ the diffusion was nearly complete, in agreement with the value of the damping constant d. Final energy distributions for the quartic and quadratic potentials are equivalent, as demonstrated analytically by Hereward [Ref. 10]. Displacements in the quartic potential are more widely spread. The bunch is longer than the bunch in the quadratic potential by approximately 2.3. Smoothed histograms are shown in Figs. 2c,d. Conoutations of longer duration or with smaller damping constant $d$ were performed showing that the asymptotic computed result for $\sigma_{\epsilon}$ is in fact $1 \mathrm{MeV}$ and that the fingl bunch lengthening factor is 1.7 .

The correct random force $N(t)$ was derived by applying the theory of filtered random processes to a system of particles in a linear harmonic potential. Then, considering the physical independence of photon emission, the same random force was used for particles in a non-harmonic potential. The next logical - step is taken in the following section. There $N(t)$ will be used for the general nonlinear systea involving time varying $R F$ fields coupled to the particle motion. That is the essential point of this discussion. The introduction of $N(t)$ into Eq. (2) is equivalent to imposing stochastic noise upon the RF potential. Rrinsky and Wang [Ref. 11] studied this problem from the viewpoint of kinetic theory using a Fokker-planck equation. 


\section{System Model}

Equations for $N$ particles in a storage ring with $M$ RF cavities are now written. The cavities are generally different one from the other and are labled sequentially by the subscript i. Associated with each cavity is a set of parameters that includes, $Q_{i}, \psi_{i}$, a coupling constant $A_{b i}$ for the current induced by the beam, a coupling constant Agi for the generator current and a phase angle for the senerator current $\eta_{\mathrm{g} i}$.

Fron Egs. (3) and (4) follow

$$
\begin{aligned}
v_{i}^{\prime}=-\left(m_{i} / 2 Q_{i}\right) v_{i} & +A_{g i} \cos \left(\eta_{i}-\eta_{g i}\right) \\
& +\frac{A_{b i}}{N} \sum_{j=1}^{N} \cos \left(\eta_{i}-m \tau_{j}\right), \\
\eta_{i}^{\prime}=\left(m_{i} / 2 Q_{i}\right) \tan \psi_{i}-\left(A_{g i} / v_{i}\right) \sin \left(\eta_{i}-\eta_{g i}\right) & -\frac{\left(A_{b i} / v_{i}\right)}{N} \sum_{j}^{N} \sin \left(\eta_{i}-m \tau_{j}\right) .
\end{aligned}
$$

- The coupling constants for the induced currents are

$$
A_{g i}=I_{g}\left(e / 2 C_{i} U_{0} \omega_{o}\right)
$$

and

$$
A_{b i}=-2 I_{b}\left(e / 2 C_{i} U_{0} \omega_{0}\right)
$$

The $N$ simulation particles included in the sums are weighted to share the average bean current $I_{b}$ equally. 
The particle equations of motion follow directly from Eqs. (1) and (2).

$$
\varepsilon_{j}^{\prime}=B\left[1-\sum_{i=1}^{M} v_{i} \cos \left(\eta_{i}-m_{i} \tau_{j}\right)\right]-d \epsilon_{j}+N_{j}(t),
$$

and

$$
\tau_{j}^{\prime}=\epsilon_{j}
$$

The sum in Eq. (13c) adds the contributions of $M$ cavities to the total RF field. The field amplitudes and phases and the particle momenta and positions constitute $2 \mathrm{M}+2 \mathrm{~N}$ state variables obeying the system model Egs. (13a,b,c,d). The parameters are externally determined constants.

Control system theory provides a compact and useful notation. A vector

$$
\bar{x}(t)=\left[\ldots, v_{i}(t), \eta \eta_{i}(t), \ldots, \epsilon_{j}(t), \tau_{j}(t), \ldots\right]
$$

is written to represent the state of the system. Another vector

$$
\overline{\mathbf{a}}=\left[\ldots, Q_{i}, \psi_{i}, A_{b_{i}}, A_{g i}, \eta_{g i}, \ldots\right]
$$

contains values of the parameters used to control the system. Simultaneously integrating all of Eqs. ( $13 a, b, c, d)$ between fixed time linits gives the state at $t_{n+1}$ in terms of the state at an earlier time $t_{n}$. The control parameters are constant during the integration interval. At discrete points in tine the control parameters may be altered. These changes are executed instantaneously and by a mechanism external to the system.

Si:ppose that the parameters are re-adjusted according to an algorithm that considers only their present values and the current state of the systen. Then the system motion may be described by a pair of iterative relations,

$$
\begin{aligned}
\bar{a}_{n} & =\bar{a}\left\{x(t), a_{n-1}\right\}, \\
\bar{x}\left(t_{n+1}\right) & =\bar{x}\left\{x\left(t_{n}\right), a_{n}\right\} .
\end{aligned}
$$


The first relation calls upon some external intelligence to reset the control paraneters and the second propagates the state of the system foward in time by integrating its differential equations. There is no reason to assume that all the state variables are measureable. Sone of them are not. For example, the phase space coordinates of individual particles in a storage ring cannot be known. On the other hand, the amplitudes and phases of RF fields in narrow band cavities are readily measured. Control system theory has methods for treating dynamical systens when measurements are imprecise and control signals inherently noisy. They include optimal filtering and predictive estimation, often using a tool called the Ralman filter algorithm [Ref. 9]. This paper will conclude with an example involving perfect measurements and noiseless control signals. Consequently the application of Eq. (14a) will involve only the state variables of the RF field and in Eq. (14b), the control parameters will not fluctuate. 


\section{An Example}

In this section Eqs. $(13 a, b, c, d)$ and $(14 a, b)$ are applied to a storage ring with a harmonic cavity RF systen. The numerical computations use data that closely describe features of the NSLS VUV ring. They are not intended to represent the operation or design of that facility, here taken as an example having a high current beam that loads its cavities heavily. All of the data needed for this simulation are listed in Appendix II.

System states will be computed from from an initial state at $t=0$, when the beam current is still zero, to a final state when the bean current reaches about one ampere. For all $t$ less than zero the generator current in the fundamental cavity and the generator current in the fourth harmonic cavity support $a$ guartic potential according to example (II) in section 4 above. Beginning at $t=0$ the bean current rises slowly toward its maximium value. The anplitude and phase of the field in each cavity are forced to change and the system cannot maintain the quartic potential without intervention. The RF generator parameters $I_{g}$ and $\eta g$ and no other parameters will be
adjusted.

To use the parameter iteration relation Eg. (14a) an algorithm is needed for measuring the fields and correcting $I_{g}$ and $\eta_{\mathrm{g}}$. The algorithm described now has been implemented in the simulation. It is practical for application in a physical control system. In the algorithm initial RF voltages and phases are recorded as a complex number for each cavity. Let $Z_{0}$ represent the voltage of one cavity at $t=0$. Then $Z_{0}$ is a target value for control system operations attempting to preserve the initial RF potential in the face of varying loading by the beam. Measurements and corrections are made periodically at times $t_{n}$. The instantaneous voltage at $t=t_{n}$ is equal to the vector sum of a part driven by the generator current and a part driven by the beam.

$$
z_{n}=z_{n}^{g}+z_{n}^{b} \text {. }
$$

Subtracting the error $\Delta z_{n}=z_{n}-z_{0}$ from $z_{n}^{g}$ corrects the sum $Z_{n}$ to the target value $Z_{0}$. By linear superposition reducing one part by the error is equivalent to correcting the sum. When $I_{g}$ and $\eta_{g}$ are changed $Z_{n}^{g}$ will respond by approaching a steady state at a rate determined by the cavity $Q$. I $g$ and $\eta g$ are determined so that the new generator part of the steady state field will be less than the old one by exactly the error $\Delta Z_{n}$. 
The algorithm is completed by relating the control paraneters to the field amplitude and phase in the steady state. Assuming that all of the particles relax to an equilibrium state with $\epsilon^{\prime}=\epsilon=\tau=0$, the beam can be considered to behave like a single rigid bunch. Neglecting quantum excitation Egs. (13c,d) reduce to average energy conservation. Eqs $(13 a, b)$ have steady state solutions of the Porm

$$
\begin{aligned}
V / 2 Q & =A_{b} \cos \eta+A_{g} \cos \left(\eta-\eta \eta_{g},\right. \\
(V / 2 Q) \tan \psi & =A_{b} \sin \eta+A_{g} \sin \left(\eta-\eta \eta_{g}\right) .
\end{aligned}
$$

Solving for $V$ and $\eta$ gives the amplitude and phase of the complex voltage $Z$.

$$
\begin{aligned}
V= & 2 Q \cos \psi\left[A_{b}^{2}+A_{g}^{2}+2 A_{b} A_{g} \cos \eta_{g}\right]^{\frac{1}{2}} . \\
\cos \eta= & {\left[\left(A_{b}+A_{g} \cos \eta_{g}\right) \cos \psi-A_{g} \sin \eta_{g} \sin \psi\right] * } \\
& {\left[A_{b}^{2}+A_{g}^{2}+2 A_{b} A_{g} \cos \eta\right]_{g}^{-\frac{1}{2}} . }
\end{aligned}
$$

Corresponding expressions for the individual components $z^{b}$ and $\mathrm{Z}^{9}$ follow by suppressing either $\mathrm{Ag}_{\mathrm{g}}$ or $\mathrm{A}_{\mathrm{b}}$.

In reality the system is dominated by non-steady state effects. For example, by filling the ring the average bean current grows and the cavity loading is gradually increased. The filling rate is generally so slow that the associated transients are not significant. However, as the beam current grows so does the coupling between the cavity modes and synchrotron oscillations, forcing perpetual transient effects. As a result, measurements of $Z$ will not necessarily represent steady states. Even so, if the control system suppresses fluctuations to acceptable levels then it is a success.

The most difficult problem of the double cavity system is instability of the synchrotron mode coupled to the harmonic cavity. It was successfully suppressed in the simulation. Low level fluctuations of field amplitude and phase were achieved in both cavities giving satisfactory bunch lengthening in a 32 pseudo-particle simulation of a 1 Ampere beam. Because the beam current was high, the fundamental cavity detuning angle 
was -89.5 degrees and the fourth harmonic cavity was detuned to +87.0 degrees. This positive detuning, which is required to achieve a quartic potential, is the source of instability. Luckily the correction algorithn neutralizes it. This effect has not yet been derived by a linear analysis of the control system but it was unerringly observed in the numerical simulation. The aleorithm corrections have exactly the opposite effect for the fundamental cavity. They tend to to cancel the stability afforded by the negative detuning. Therefore under-correction factors were introduced. In the successful simulation runs the fundamental cavity correction was reduced by a factor 0.25. For the harnonic cavity it was reduced by 0.9 . There was some interaction between optimal values for these factors and the time interval between control systen iterations.

A computation was performed extending to $5 \times 10^{6}$ radian units of time. Fig. 3 shows the amplitude and phase of the field in each cavity during the first $5 \times 10^{5}$ radians. Fig. 4

shows them diring the last $5 \times 10^{5}$ radians. To save computation time, the risetime of the beam current was artificially

shortened to $5 \times 10^{4}$ radians. This is much faster than in actual storage rings, yet slower than the time for a cavity field to adiabatically respond. RE system corrections were made every

$2.5 \times 10^{4}$ radians. Since the particle diffusion time constant was $2 \times 10^{6}$ radians the bunch length at the end of the computation would represent, apart fron residual fluctuations, a steady state result.

Statistical averages for the 32 pseudo-particles at the end of the simulation run are shown in Table I. The average particle displacement is very close to the synchronous position. Also, the average particle energy is very close to Eo. The bunch length is longer than computed for the equivalent fixed quartic potential, the energy standard deviation $\sigma_{\epsilon}$ is less than $1 \mathrm{MeV}$, and the correlation between $\epsilon$ and $\tau$ is small. By implication, field fluctuations are small enough that the particles fill a nearly stationary potential well to a level far above its perturbed bottom. Any sloshing of the potential well, or trapping of particles at a certain phase in it, would occur below the thermal energy limit. The fluctuations probably do contribute to the longer bunch length while the added dissipation from the cavity losses may tend to cool the beam. 


\section{Conclusion}

Harmonic cavity bunch lengthening poses challenging problems in nonlinear particle dynamics, electrodynamics and accelerator system control. The method described in this report reduces this problem to a system model consisting of coupled, first order, nonlinear differential equations for the particles and the fields. Taking advantage of large differences in the physical time scales involved, it is possible to separate fast and slow phenomena to write equations for the slow evolution of the state of the complete system in the format used in control system theory.

An advantage of the state variable format is the ease of introducing stochastic effects. The dominant stochastic effect in storage rings, quantum excitation, was successfully included. The computation of the model was then expanded to include discrete time $R F$ control system corrections. Opportunities for further expansion are numeraus. For example, stochasticity due to imperfect $R$ field measurement can be added and at a higher level of sophistication it should be possible to apply the theory of predictive estimation [Re?. 9] to determine optimal parameter adjustments.

What about other well known phenomena in storage $r$ ing dynamics? By simply introducing equations for an idler cavity and using several rigid particle bunches, coupled bunch instabilties may be computed from an initial perturbation to their assymptotic saturation. By starting with equations for betatron motion it should also be possible to build a state variable model for the transverse motion. 


\section{Table I}

$$
\begin{aligned}
& \langle\varepsilon\rangle=-0.0302 \mathrm{MeV} \quad\langle\gamma\rangle=0.0263 \text { Radians } \\
& \sigma_{\epsilon}=0.7986 \mathrm{MeV} \quad \sigma_{\tau}=0.2606 \text { Radians } \\
& R_{E T}=0.0498
\end{aligned}
$$


References

1. J. Le Duff, "Current and Current Density Limitations in Existing Electron Storage Rings", Nucl. Instr. Meth. in Phys. Res. A239 (1985) pp. 83-101.

2. A. Hofmann and S. Myers, "Beam Dynamics in a Double RF System", Proceedings 11 th Int. Conf. on High Energy Accelerators, CERN (1980), Pp. 610-614.

3. Gudfrey Saxon, "Harmonic RE System", European Synchrotron Radiation Project Internal Report ESRP-IRH-69/84, September 1984 .

4. This report describes a continuation of earlier work at the National Synchrotron Light Source, Brookhaven National Laboratory. It supercedes J.M. Wachtel, "On Bunch Lengthening Using the Eourth Harmonic Cavity in the NSLS VUV Ring", BNL 40929, February 1988.

5. S. Rrinsky and J. M. Wang, "Longitudinal Instabilities of Bunched Beams Subject to a Non-Harmonic RE Potential", Particle Accelerators, 1985, Vol. 17, pp. 109-139.

6. Samuel Krinsky, "Saturation of a Longitudinal Instability due to Nonlinearity of the Wake Field", Brookhaven National Laboratory Report BNL 36430, 1985.

7. M. Sands, "The Physics of Electron Storage Rings: An Introduction", in International School of Physics Enrico Fermi, Academic Press, 1971, pp. 257-411.

8. Y. Miyahara, S. Asaoka, A. Mikuni, and K. Soda, "Equilibrium Phase Instability in the Double Re System for Landau Damping", Nucl. Instr. Meth. in Phys. Res. A260 (1987) pp. 518-528.

9. Robert Grover Brown, "Randon Signal Analysis and Ralman Filtering", John Wiley and Sons (1983).

10. H.G. Hereward, "Equilibrium Energy Distribution in a NonLinear Potential Well in the Presence of Quantum Fluctuations", PEP Summer Study Note 53, August 1973.

11. S. Rrinsky and J.M. Wang, "Bunch Diffusion due to RF Noise", Particle Accelerators, 1982, Vol. 12, pp. 107-117. 
App endix I

Equations governing the slow time behavior of the fields in a narrow band cavity are now derived. The cavity is a single mode, capacitively loaded resonator with an axisymmetric gap. It is excited by a bean crossing the gap and also by a coupled RF generator.

Using a vector potential $\bar{A}(\bar{r}, t)$ in the radiation gauge having boundary conditions appropriate to the cavity mode,

$$
\begin{gathered}
\overline{\bar{E}}=-\partial \bar{A} / \partial \mathrm{t}, \\
\left(\omega_{0}+\Delta \omega\right)^{2} \bar{A}+\partial^{2} \bar{A} / \partial t^{2}=\mu_{0} c^{2} \bar{J},
\end{gathered}
$$

where $\omega_{0}$ is the RF generator frequency, $\omega_{0}+\lambda \omega$ is the resonant frequency of the cavity and $J$ is the current density anywhere within its boundaries including the beam current and also the generator current referred to an equivalent location in the cavity circuit. The boundary includes the volume penetrated by field in imperfectly conducting walls. The RF enerator frequency is equal to the nearest harmonic of the current in a synchronous beam to the cavity resonant frequency. written

Assuming a tenuous beam, the vector potential may be

$$
\bar{A}(\bar{r}, t)=a(t) \sin \left[\omega_{0} t+\eta(t)\right] \bar{u}(\bar{r})
$$

where $\bar{u}(r)$ is the empty cavity spatial mode, $a(t)$ is a slowly varying amplitude and $\eta(t)$ is a slowly varying phase. Slowly

varying means that $\dot{a} \ll \omega_{0}$ and $\dot{\eta} \ll \omega_{0}$.

Substituting Eq. (A3) into Eq. (A2) and separating quadrature components by averaging over time leads to two equations

$$
\begin{array}{r}
2 \dot{a}\left(\omega_{0}+\dot{\eta}-\Delta \omega\right)+a \ddot{\eta}=2 \mu_{0} c^{2}\left\langle J_{c}\right\rangle, \\
\ddot{a}-2 a \omega_{0}(\dot{\eta}-\Delta \omega)-a(\dot{\eta}-\Delta \omega)^{2}=2 \mu_{0} c^{2}\left\langle J_{s}\right\rangle,
\end{array}
$$


where

$$
\left\langle J_{S, C}\right\rangle=\left[2 T \int u^{2} d^{3} r\right]^{-1} \int_{-T}^{T} d t \cos ^{\sin }\left[\omega_{\delta} t+\eta\right] \int d^{3} r \bar{u}(\bar{r}) \cdot \bar{J}(\bar{r}, t) .
$$

The volume of integration includes the part of the wall penetrated by RF current and field. The time interval $\mathrm{T} \gg \omega_{0}^{-1}$ but it is sufficiently short that $a(t)$ and $\eta(t)$ are unchanged. Retaining terms to first order in $\omega_{0}^{-1}$, Eqs. (A4a,b) simplify to

and

$$
\dot{a}=\mu_{0} c^{2} \omega_{0}^{-1}\left\langle J_{c}\right\rangle
$$

$$
a(\eta) \Delta \omega)=-\mu_{0} c^{2} \omega_{0}^{-1}\left\langle J_{S}\right\rangle \text {. }
$$

The corresponding lowest order approximation for the electric field is

$$
\bar{E}(\bar{r}, t)=-\omega_{0} a(t) \bar{u}(\bar{r}) \cos \left[\omega_{0} t+\eta(t)\right] .
$$

In a typical storage ring RF cavity the bean traverses a short gap where the RF electric field is large and the magnetic field is small. Consider a cavity having a short gap of length $\triangle z$ located at the origin of coordinates $r=0$. By Eq. (AG) the gap potential is

$$
-E(0, t) \Delta z=v(t) \cos \left[\omega_{0} t+\eta\right],
$$

where

$$
v(t)=\omega_{0} \bar{u}(0) \cdot \overrightarrow{\Delta z} \mathbf{a}(t) .
$$

It is convenient to define a capacitance

$$
c=\frac{\epsilon_{0}}{u^{2}(0)(\Delta z)^{2}} \int d^{3} r u^{2}(r)
$$

such that the stored RF energy is equal to $(1 / 2) \mathrm{Cv}^{2}$. For a cavity with heavy capacitive loading at the gap, $C$ is essentially equal to the capacitance between the adjacent boundaries. 
There are three distinct contributions to the current density $J(r, t)$. First there is the current due to the bean which may be considered as the sum of charges moving on a line. Next there is the drive current due to a coupled RF generator. It is convenient to refer this current to the gap as if the coupling port were located there. Finally there are the currents induced in the cavity wall, repesenting pure loss as they oscillate in phase with the local electric field and also the current induced in the coupled generator impedance which may have a reactive component as well. Writing the current density

$$
\bar{J}(r, t)=\hat{z}\left[I_{b}(t)+I_{g}(t)\right] \delta(x) \delta(y)+\bar{J}_{w},
$$

and using it in Eqs (A5a,b) leads to

$$
\dot{v}+\left(\omega_{0} / 2 Q\right) v=(2 C T)^{-1} \int_{-T}^{T}\left[I_{b}(t)+I_{g}(t)\right] \cos \left[\omega_{0} t+\eta\right] d t
$$

and

$$
v(\dot{\eta}-\Delta \omega)=-(2 \mathrm{CT})^{-1} \int_{-T}^{T}\left[I_{b}(t)+I_{g}(t)\right] \sin \left[\omega_{0} t+\eta\right] d t \text {. }
$$

The term involving $Q$ includes the resistive loss in the cavity wall and the resistive part of the coupled generator impedance. while the coupled reactance is included in the detuning term $\Delta \omega$.

Particles cross the cavity gap at times $t=j T_{0}-\tau(t)$.

Therefore the current in the gap due to the ith electron on its jth revolution may be written $e \delta\left(t+\tau_{i}(t)-j T_{0}\right)$. Summing over all electrons and all revolutions,

$$
I(t)=\sum_{i, j} e \delta\left(t+\tau_{i}(t)-j T_{0}\right) .
$$


It follows that

$$
(2 T)^{-1} \int_{b^{(}(t)}^{T} \cos \left[\omega_{0} t+\eta\right] d t=\left(e / T_{0}\right) \sum_{i} \sin \left[-\omega_{0} \tau_{i}+\eta\right] .
$$

Since the generator current is a pure harmonic of the rotation frequency,

$$
I_{g}(t)=I_{g} \cos \left(\omega_{0} t+\eta_{g}\right)
$$

and the corresponding integral for the generator current is $(1 / 2) \operatorname{Ig}_{\cos }^{\sin }(\eta-\eta / g)$. Substitution in Eqs. $(A 7 a, b)$ leads directly to Eqs. ( 3 ) and (4) in section 2 . 
Numerical computations are based upon the parameters of a 9 bucket storage ring with two RF cavities, a fundamental cavity and a fourth harmonic cavity.

$\begin{array}{lcc}m & 1 & 4 \\ f(K H z) & 52.88 & 211.52 \\ Q & 20,000 & 4,000 \\ C(p f d) & 64 & 38 \\ R_{S}(K \Omega) & 940 & 79\end{array}$

Values for $Q$ and the equivalent shunt impedance include the coupled generator impedance.

The storage ring parameters needed for computation are the synchronous particle energy, the average radiation loss per revolution, the critical photon energy, the compaction factor and the particle coherent damping constant.

$$
\begin{aligned}
E_{0} & =0.75 \mathrm{GeV} \\
U_{0} & =14.0 \mathrm{keV} \\
u_{c} & =1.0 \mathrm{keV} \\
\alpha & =0.02 \\
d & =220 \mathrm{sec}^{-1} \\
& =6.6 \times 10^{-1} \text { radians at } 52.88 \mathrm{MHz} .
\end{aligned}
$$

The $52.88 \mathrm{HHz}$ RF generator is initially set for $77 \mathrm{kgV}$ peak $R F$ voltage at the fundamental cavity gap or $e V_{1}=5.5 U_{0}$. 


\section{Figure Captions}

Fig. 1. Normalized mode eigenvalues $s_{i} / s_{0}$ are plotted in the complex plane for increasing values of the bean-cavity coupling constant $A Q$. Imaginary values represent the mode frequencies and real values $g$ ive the rate of exponential damping or growth. The nomalized cavity damping factor $u=m / 2 Q s_{0}$ is 1 and the cavity detuning angle $\psi$ is $0.4 \pi$ in (a) and $-0.4 \pi$ in (b). The unstable modes in (a) are synchrotron oscillations of growing anplitude below a threshold value of $A Q$. Above it they show monotone growth.

Fig. 2. Distributions created by smoothing 32 bin histograms of the phase space data for 512 particles are shown above. Results for the quadratic potential are labled I and for the quartic potential they are labled II. The data in (a) and (b) are for elapsed time $T=5 \times 10^{5}$ radians and in (c) and (d) for $T=2 \times 10^{6}$ radians when the diffusion process is nearly complete. To the accuracy limited by the sample size, the energy distributions in (d) are indistinguishable.

Fig. 3. Amplitude and phase of the RF field at the beginning of a 32 pseudo-particle simulation. The initial field variables satisfy the conditions for a quartic potential bucket. Generator parameters are corrected every $2.5 \times 10^{4}$ radians.

Fig. 4. Amplitude and phase of the RF field when the system has approached its final state. Fluctuations around the initial field variable values persist. 


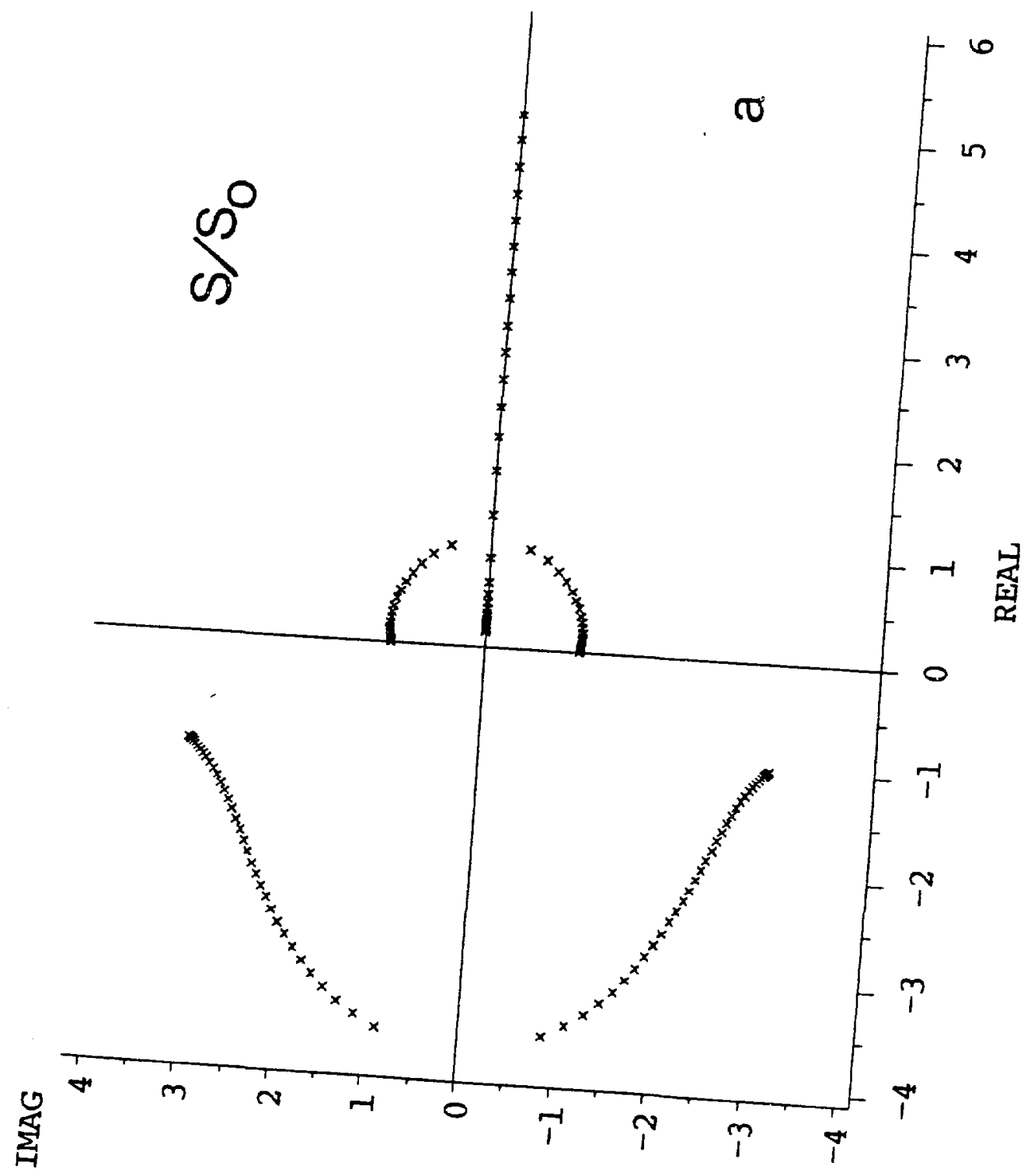




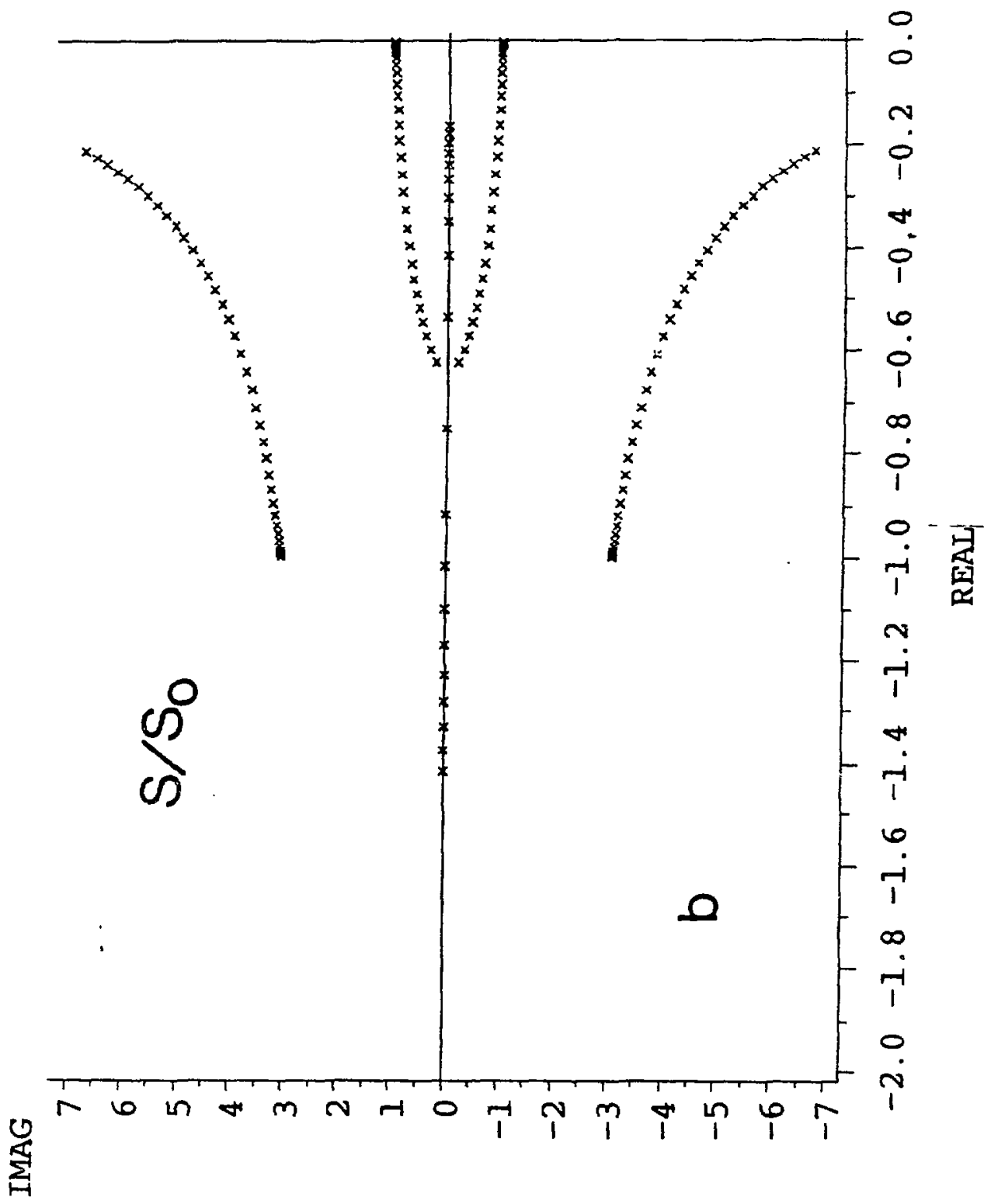


Fig $2 a, b$
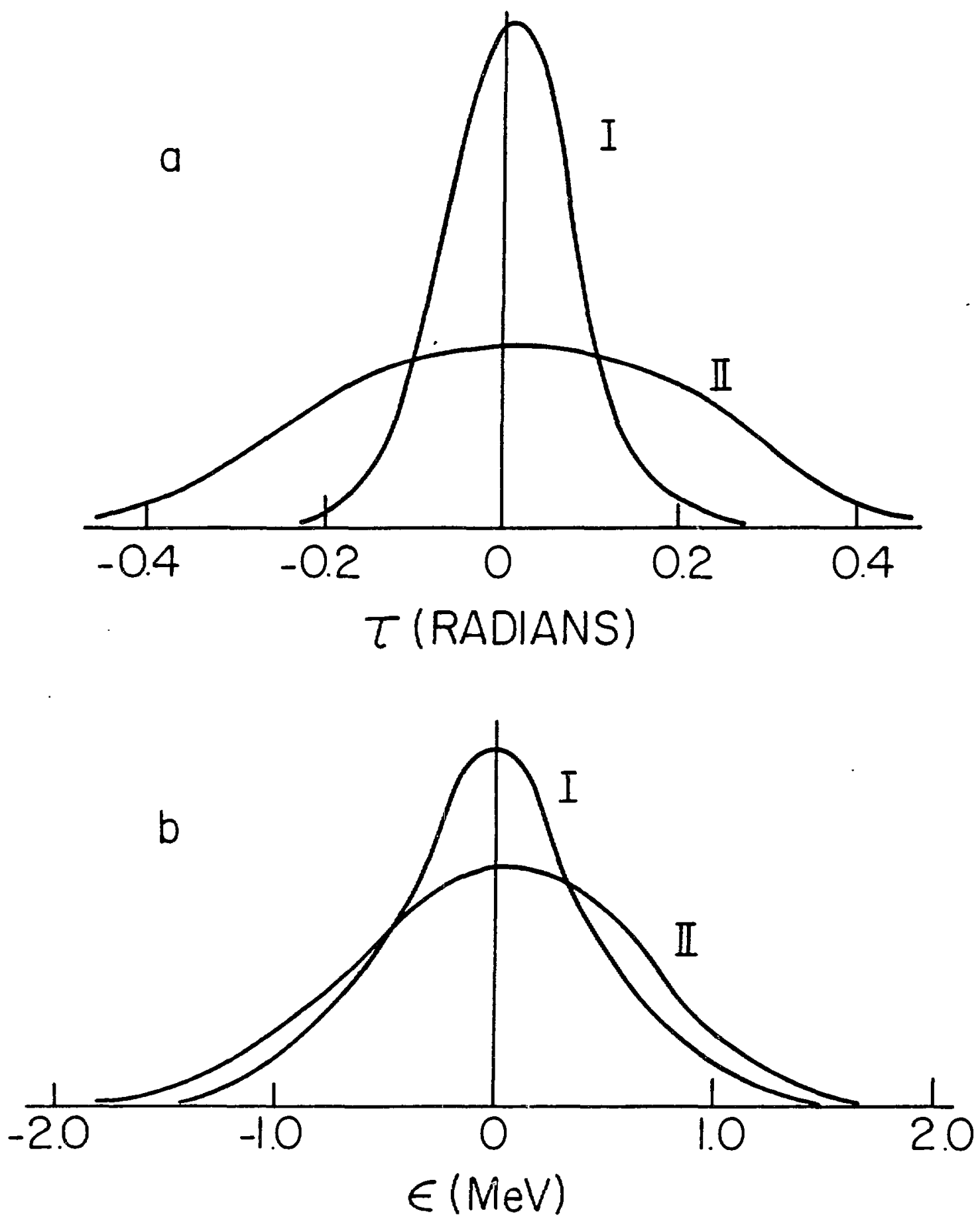
Fig $2 c, d$
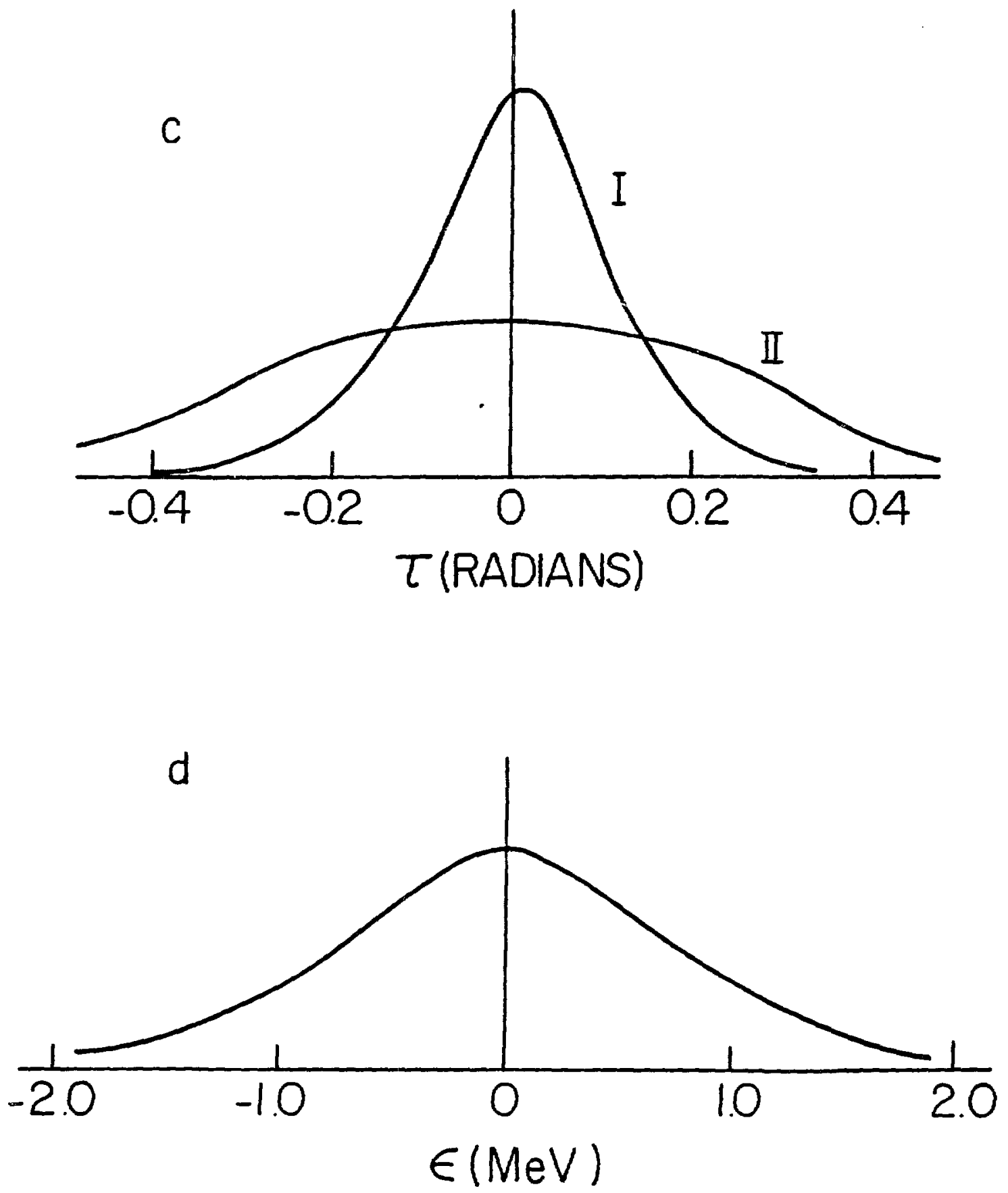
Fig $3 a, b$
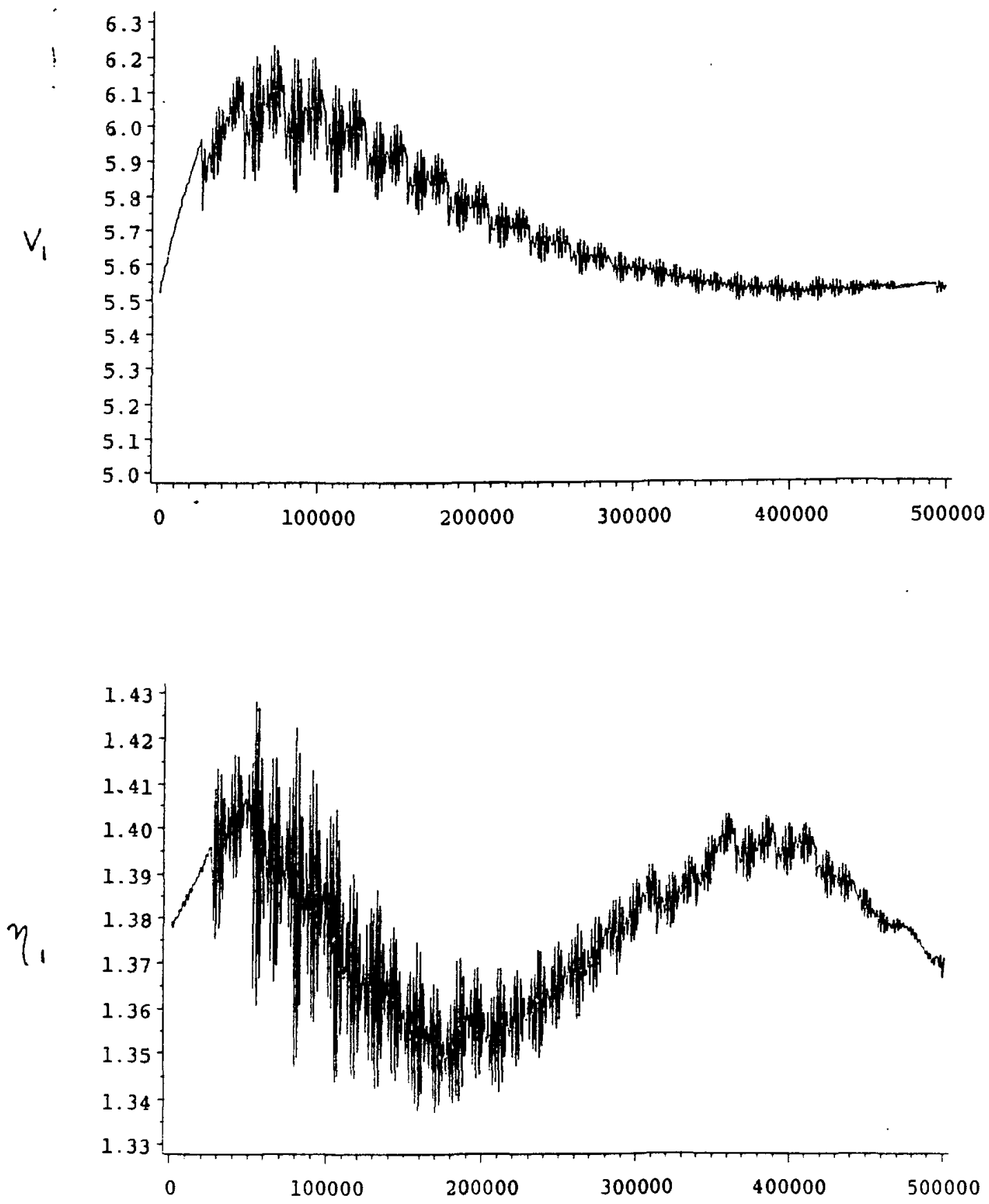
Fig $3 c, d$
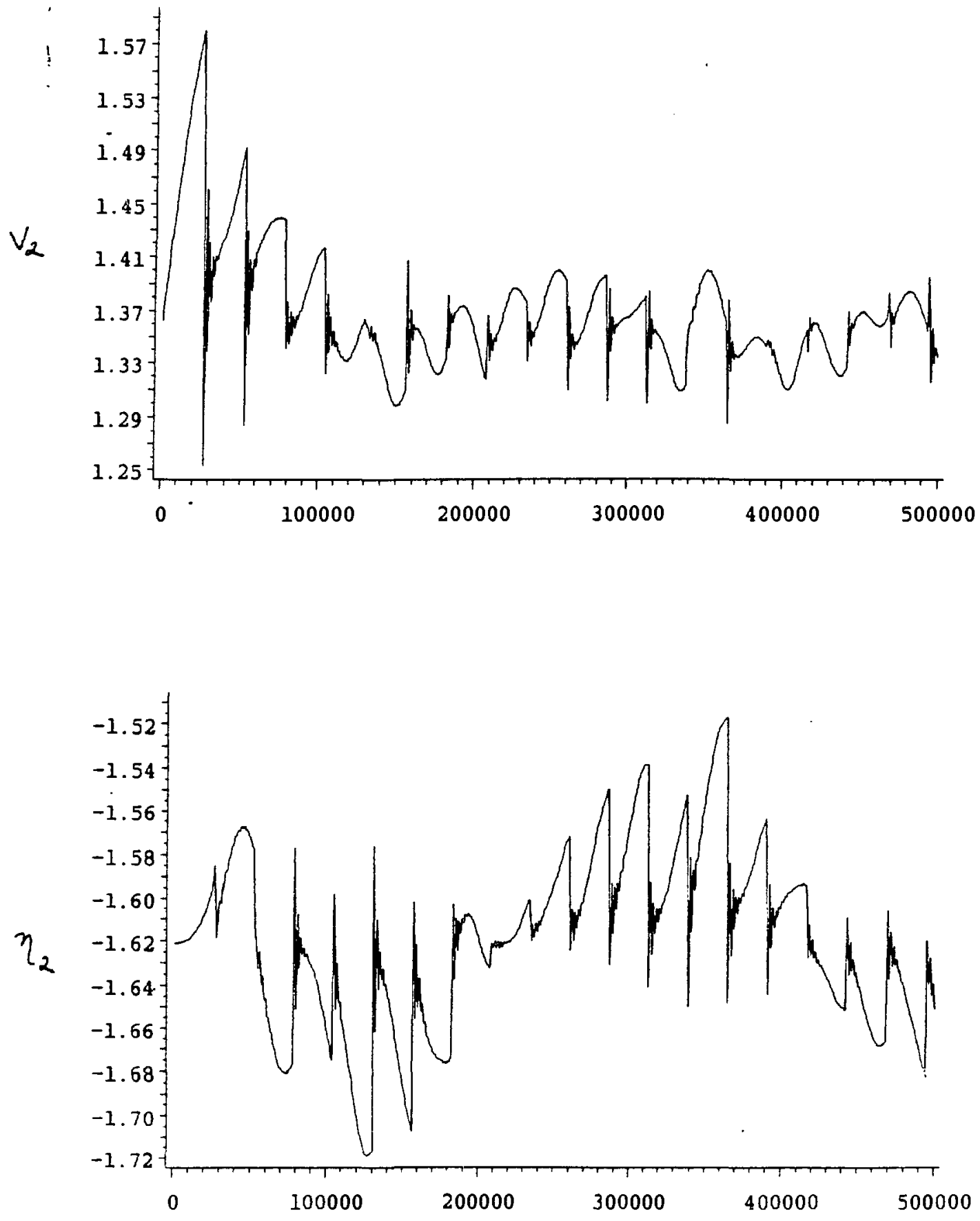
Fig $4 a, b$
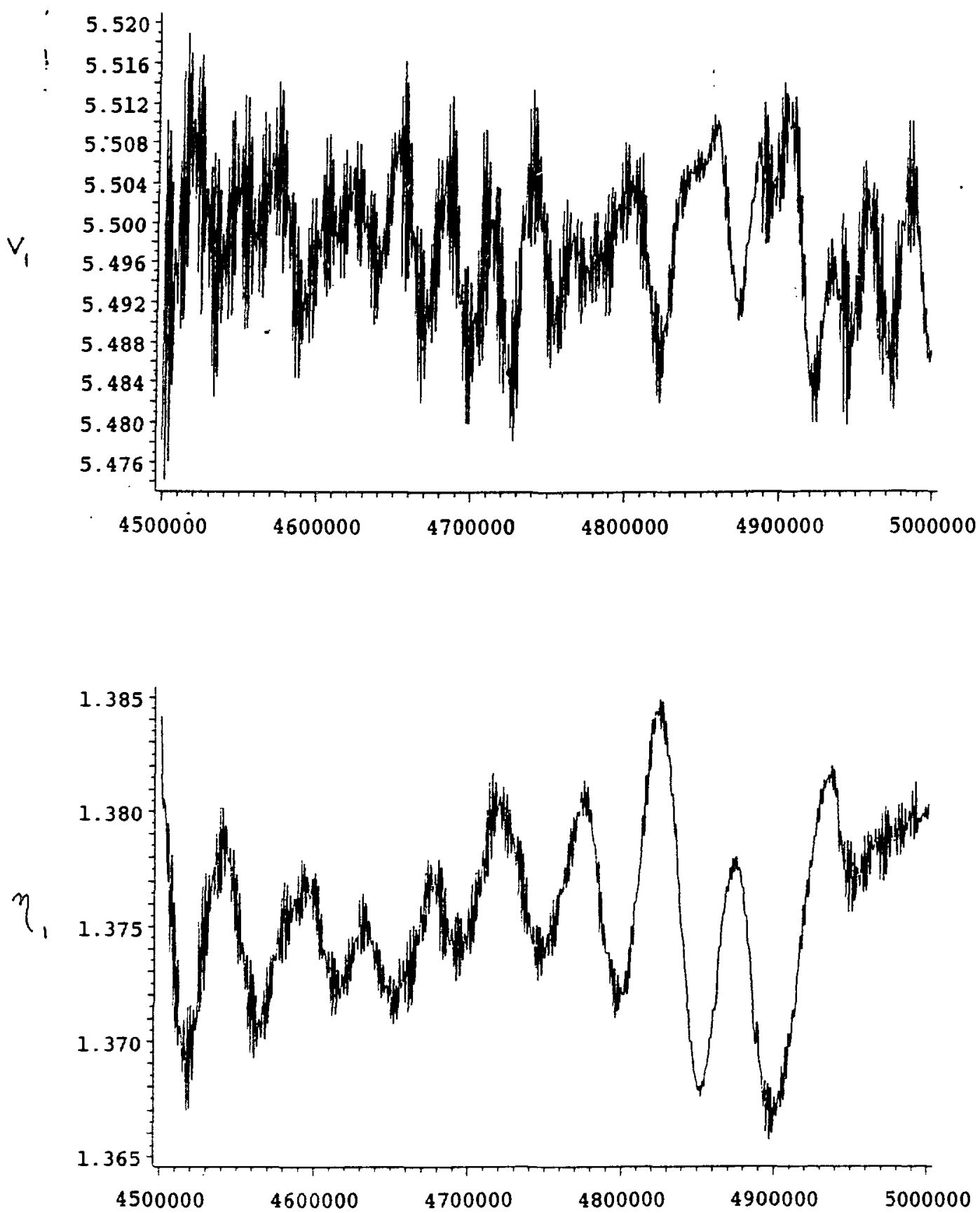
Fig $4 c, \alpha$
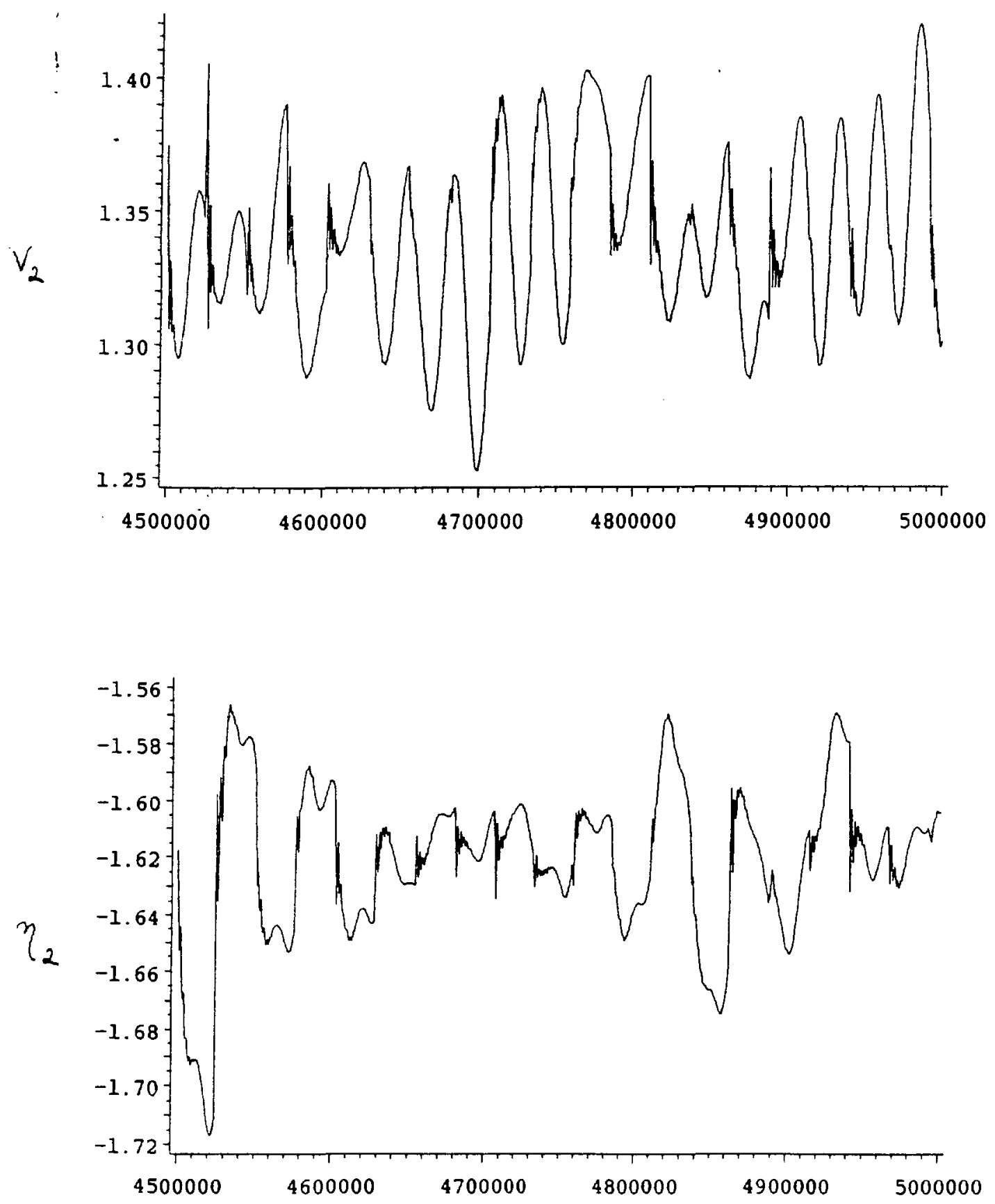